Submitted to the Annals of Statistics

\title{
SUPPLEMENTARY MATERIAL: THE ASYMPTOTICS OF RANKING ALGORITHMS
}

\author{
By John C. Duchi , Lester Mackey and Michael I. Jordan \\ University of California, Berkeley and Stanford University
}

In this supplement, we provide the proofs of our main results, their corollaries, and supporting lemmas.

\section{Proofs of Consistency Results.}

8.1. Proof of Proposition 1. We begin by recalling the definition of the convex conjugate $h^{*}$ of a function $h$ and its biconjugate $h^{* *}$

$$
h^{*}(y):=\sup _{x}\{y x-h(x)\} \quad \text { and } \quad h^{* *}(x):=\sup _{y}\left\{x y-h^{*}(y)\right\}
$$

noting that $h^{* *}(x) \leq h(x)$ and that $h^{* *}$ is closed convex [4]. The following lemma is useful for the proof of Proposition 1.

Lemma 2. Let $h:[0,1] \rightarrow \mathbb{R}_{+} \cup\{\infty\}$ be non-decreasing with $h(0)=0$ and $h(x)>0$ for $x>0$. Then $h^{* *}(x)>0$ for $x>0$.

Proof. For the sake of contradiction assume that there is some $z \in(0,1)$ with $h^{* *}(z)=0$. Since $h^{* *}$ is convex with $h^{* *}(0)=0$, we have $h^{* *}([0, z])=$ $\{0\}$, and in particular $h^{* *}(z / 2)=0$. By assumption $h(z / 2)=b>0$, whence $h(1) \geq b>0$. Thus we can define the closed convex function

$$
g(x)= \begin{cases}0 & \text { if } x \leq z / 2 \\ \frac{b}{1-z / 2}(x-z / 2) & \text { otherwise }\end{cases}
$$

and have $g(x) \leq h(x)$ since $h(1) \geq h(z / 2)=b>0$. It is clear, however, that $g(z)>0=h^{* *}(z)$, which contradicts the fact [4] that $h^{* *}$ is the greatest closed convex minorant of $h$.

Proof of Proposition 1 The proof is analogous to proofs of similar results due to Zhang [11], Tewari and Bartlett [9], and Steinwart [8]. First, note that the function $H$ is non-decreasing in its argument and satisfies 
$H(0)=0$ and $H(1+\epsilon)=\infty$ for all $\epsilon>0$. Jensen's inequality implies that

$$
\begin{aligned}
H^{* *}\left(R(f)-R^{*}\right) & =H^{* *}\left(\sum_{q} p_{q}\left[\ell\left(f(q), \mu_{q}\right)-\inf _{\alpha} \ell\left(\alpha, \mu_{q}\right)\right]\right) \\
& \leq \sum_{q} p_{q} H^{* *}\left(\ell\left(f(q), \mu_{q}\right)-\inf _{\alpha} \ell\left(\alpha, \mu_{q}\right)\right) \\
& \leq \sum_{q} p_{q} H\left(\ell\left(f(q), \mu_{q}\right)-\inf _{\alpha} \ell\left(\alpha, \mu_{q}\right)\right) \\
& \leq \sum_{q} p_{q}\left(\ell_{\varphi}\left(f(q), \mu_{q}\right)-\inf _{\alpha} \ell_{\varphi}\left(\alpha, \mu_{q}\right)\right) \\
& =R_{\varphi}(f)-R_{\varphi}^{*},
\end{aligned}
$$

the final inequality following by the definition (8) of $H$. Lemma 2 implies that $H^{* *}(\epsilon)>0$ for $\epsilon>0$, and since $H^{* *}$ is closed convex and non-negative, it is continuous from the right at 0 so long as $H^{* *}(\epsilon)<\infty$ for some $\epsilon>0$.

Now, assume that $\varphi$ is pointwise consistent. Let $f_{n}$ be a sequence of measurable functions such that $R_{\varphi}\left(f_{n}\right) \rightarrow R_{\varphi}^{*}$. For shorthand, define $h(\epsilon)=$ $H^{* *}(\epsilon)$. If $H^{* *}(\epsilon)=\infty$ for all $\epsilon>0$, then $H(\epsilon) \geq H^{* *}(\epsilon)=\infty$ for all $\epsilon>0$. Then the definition (8) and the finiteness $R_{\varphi}(f)<\infty$ imply that $\ell(f(q), \mu)-\inf _{\alpha^{\prime}} \ell\left(\alpha^{\prime}, \mu\right)=0$ for all $\mu \in \mathcal{M}(\mathcal{S})$, and hence $\delta(\epsilon)=\infty$ in Definition 2.

Thus we can assume that $H(\epsilon)<\infty$ for some $\epsilon>0$, and let $h_{\leftarrow}^{\prime}(\epsilon)$ denote the right-derivative of $h$ at the point $\epsilon$ for $\epsilon \in \operatorname{int} \operatorname{dom} h$. This interior is nonempty by assumption, and the right-derivative exists and is positive since $h$ is closed convex and $H$ is non-decreasing with $H(x)>H(0)$ for $x>0$ [4]. Now fix some $\epsilon>0$, and let $b=h_{\leftarrow}^{\prime}(\epsilon / 2)>0$. Since $h$ is convex and $b$ is a subgradient of $h$, we have $h(\epsilon) \geq h(\epsilon / 2)+b \epsilon / 2>b \epsilon / 2$, so that if $h(\delta)<b \epsilon / 2$, then $\delta<\epsilon$. Applying the bound (1), we can take $N \in \mathbb{N}$ such that $n \geq N$ implies $R_{\varphi}\left(f_{n}\right)-R_{\varphi}^{*}<b \epsilon / 2$, and as such $H^{* *}\left(R\left(f_{n}\right)-R^{*}\right)<b \epsilon / 2$ so that $R\left(f_{n}\right)-R^{*}<\epsilon$.

Now we turn to the converse, where we assume that $\varphi$ is uniformly consistent. Let $\left\{p_{q}\right\}$ be supported completely on a single $q$. Then

$$
R(f)-R^{*}=\ell\left(f(q), \mu_{q}\right)-\inf _{\alpha^{\prime}} \ell\left(\alpha^{\prime}, \mu_{q}\right)
$$

and

$$
R_{\varphi}(f)-R_{\varphi}^{*}=\ell_{\varphi}\left(f(q), \mu_{q}\right)-\inf _{\alpha^{\prime}} \ell_{\varphi}\left(\alpha^{\prime}, \mu_{q}\right) .
$$

Fix an $\epsilon>0$, and let $\alpha=f(q) \in \mathbb{R}^{m}$. By the contrapositive of Definition 2, 
we know that there exists $\delta(\epsilon)>0$ such that

$$
\begin{gathered}
R(f)-R^{*}=\ell\left(\alpha, \mu_{q}\right)-\inf _{\alpha^{\prime}} \ell\left(\alpha^{\prime}, \mu_{q}\right) \geq \epsilon \text { implies } \\
R_{\varphi}(f)-R_{\varphi}^{*}=\ell_{\varphi}\left(\alpha, \mu_{q}\right)-\inf _{\alpha^{\prime}} \ell_{\varphi}\left(\alpha^{\prime}, \mu_{q}\right) \geq \delta(\epsilon),
\end{gathered}
$$

which holds for any measurable $f$ (and hence any $\alpha \in \mathbb{R}^{m}$ ). Since the measure $\mu_{q}$ was arbitrary, we see that the function $H(\epsilon)$ defined by the infimum (8) satisfies $H(\epsilon) \geq \delta(\epsilon)>0$ for all $\epsilon>0$.

8.2. Proof of Theorem 1. We prove Theorem 1 in two parts: first under Assumption $\mathrm{C}$ and second under Assumption C' under the additional condition of 0 -coercivity of $\ell_{\varphi}(\cdot, \mu)$ (recall a function $g: \mathbb{R}^{m} \rightarrow \mathbb{R}$ is called $k$-coercive [4] if $g(x) /\|x\|^{k} \rightarrow+\infty$ as $\left.\|x\| \rightarrow \infty\right)$.

The proof of Theorem 1 using Assumption $\mathrm{C}$ requires three lemmas that make it quite straightforward. We begin by recalling the definition (3) of the optimal conditional surrogate $\operatorname{loss} \ell_{\varphi}^{*}(\mu):=\inf _{\alpha} \ell_{\varphi}(\alpha, \mu)$. We have the following three results, each assuming the conditions of Theorem 1 with Assumption C.

Lemma 3 (Zhang [11], Lemma 27; Tewari and Bartlett [9], Lemma 16). The function $\ell_{\varphi}^{*}$ is continuous in the measure $\mu$.

Lemma 4. Let $\varphi$ be structure-consistent and $\left\{\alpha_{n}\right\} \subset \mathbb{R}^{m}$ be a sequence of vectors. Then $\ell_{\varphi}\left(\alpha_{n}, \mu\right) \rightarrow \ell_{\varphi}^{*}(\mu)$ implies that $\ell\left(\alpha_{n}, \mu\right) \rightarrow \inf _{\alpha} \ell(\alpha, \mu)$, and for large enough $n$, the vector $\alpha_{n} \in \operatorname{argmin}_{\alpha} \ell(\alpha, \mu)$.

Proof. Suppose for contraposition that the sequence of vectors $\left\{\alpha_{n}\right\}$ has $\ell\left(\alpha_{n}, \mu\right) \nrightarrow \rightarrow \inf _{\alpha} \ell(\alpha, \mu)$. Then there is a subsequence $n_{j}$ of $\alpha_{n}$ and some $\epsilon>0$ such that $\ell\left(\alpha_{n_{j}}, \mu\right) \geq \inf _{\alpha} \ell(\alpha, \mu)+\epsilon$ for all $j$. Thus we have $\alpha_{n_{j}} \notin$ $\operatorname{argmin}_{\alpha} \ell(\alpha, \mu)$ for any $j$, and Definition 3 implies that

$$
\ell_{\varphi}\left(\alpha_{n_{j}}, \mu\right) \geq \inf _{\alpha}\left\{\ell_{\varphi}(\alpha, \mu) \mid \alpha \notin \underset{\alpha}{\operatorname{argmin}} \ell(\alpha, \mu)\right\}>\ell_{\varphi}^{*}(\mu) .
$$

As a consequence, we have $\ell_{\varphi}\left(\alpha_{n_{j}}, \mu\right)$ uniformly bounded from $\ell_{\varphi}^{*}(\mu)$, which is the contrapositive of the lemma's first claim. Thus, whenever $\ell_{\varphi}\left(\alpha_{n}, \mu\right) \rightarrow$ $\ell_{\varphi}^{*}(\mu)$, we have $\ell\left(\alpha_{n}, \mu\right) \rightarrow \inf _{\alpha} \ell(\alpha, \mu)$, and since the range of $L$ (and hence $\ell$ by Assumption $\mathrm{C}$ ) is finite by assumption, we must have $\alpha_{n} \in$ $\operatorname{argmin}_{\alpha} \ell(\alpha, \mu)$ for sufficiently large $n$. 
Lemma 5. Let $\varphi$ be structure-consistent. Then the suboptimality function (8) satisfies $H(\epsilon)>0$ for all $\epsilon>0$.

Proof. An equivalent statement to the result that $H(\epsilon)>0$ for all $\epsilon>0$ is that for any $\epsilon>0$, there exists a $\delta(\epsilon)>0$ such that for any $\mu \in \mathcal{M}(\mathcal{S})$,

$$
\ell(\alpha, \mu)-\inf _{\alpha^{\prime}} \ell\left(\alpha^{\prime}, \mu\right) \geq \epsilon \text { implies } \ell_{\varphi}(\alpha, \mu)-\ell_{\varphi}^{*}(\mu) \geq \delta(\epsilon) .
$$

We give a proof by contradiction using Lemmas 3 and 4 . Assume that there is a sequence of pairs $\left(\alpha_{n}, \mu^{n}\right) \in \mathbb{R}^{m} \times \mathcal{M}(\mathcal{S})$ with

$$
\ell\left(\alpha_{n}, \mu^{n}\right)-\inf _{\alpha^{\prime}} \ell\left(\alpha^{\prime}, \mu^{n}\right) \geq \epsilon \quad \text { and } \quad \ell_{\varphi}\left(\alpha_{n}, \mu^{n}\right)-\ell_{\varphi}^{*}\left(\mu^{n}\right) \rightarrow 0 .
$$

By the compactness of $\mathcal{M}(\mathcal{S})$ (recall $\mathcal{S}$ is finite), we can take a subsequence $n_{j}$ such that $\mu^{n_{j}} \rightarrow \mu$ for some $\mu \in \mathcal{M}(\mathcal{S})$. Then Lemma 3 gives $\ell_{\varphi}^{*}\left(\mu^{n_{j}}\right) \rightarrow$ $\ell_{\varphi}^{*}(\mu)$; this coupled with the fact that $\ell_{\varphi}\left(\alpha_{n}, \mu^{n}\right)-\ell_{\varphi}^{*}\left(\mu^{n}\right) \rightarrow 0$ implies

$$
\lim _{j} \ell_{\varphi}\left(\alpha_{n_{j}}, \mu^{n_{j}}\right)=\lim _{j} \ell_{\varphi}^{*}\left(\mu^{n_{j}}\right)=\ell_{\varphi}^{*}(\mu) .
$$

If we can show that $\lim _{j} \ell_{\varphi}\left(\alpha_{n_{j}}, \mu\right)=\ell_{\varphi}^{*}(\mu)$, then we are done. Indeed, if this is the case then Lemma 4 implies that $\alpha_{n_{j}} \in \operatorname{argmin}_{\alpha} \ell(\alpha, \mu)$ eventually, whence $\ell\left(\alpha_{n_{j}}, \mu\right)-\inf _{\alpha^{\prime}} \ell\left(\alpha^{\prime}, \mu\right)=0$. Then the continuity of $\ell(\alpha, \mu)$ in $\mu$ contradicts $\ell\left(\alpha_{n}, \mu^{n}\right)-\inf _{\alpha^{\prime}} \ell\left(\alpha^{\prime}, \mu^{n}\right) \geq \epsilon$ for all $n$.

To the end of showing $\lim _{j} \ell_{\varphi}\left(\alpha_{n_{j}}, \mu\right)=\ell_{\varphi}^{*}(\mu)$, fix an arbitrary $\epsilon^{\prime}>0$ and recalling that $\mathcal{S}$ is finite, let $\mathcal{S}_{+}$denote the set of $s \in \mathcal{S}$ such that $\mu(s)>0$. Since $\mu^{n_{j}} \rightarrow \mu$, there must be some $B \in \mathbb{R}$ and $J \in \mathbb{N}$ such that $\varphi\left(\alpha_{n_{j}}, s\right) \leq B$ for all $j \geq J$ and $s \in \mathcal{S}_{+}$. Now, choose $J^{\prime} \geq J$ so that $j \geq J^{\prime}$ implies $\mu(s) \leq \mu^{n_{j}}(s)+\epsilon^{\prime} / B|\mathcal{S}|$. Then

$$
\int_{\mathcal{S}_{+}} \varphi\left(\alpha_{n_{j}}, s\right) d \mu(s) \leq \int_{\mathcal{S}_{+}} \varphi\left(\alpha_{n_{j}}, s\right) d \mu^{n_{j}}(s)+B|\mathcal{S}| \cdot \frac{\epsilon^{\prime}}{B|\mathcal{S}|}
$$

for $j \geq J^{\prime}$, and thus

$$
\begin{aligned}
\limsup _{j} \ell_{\varphi}\left(\alpha_{n_{j}}, \mu\right) & =\limsup _{j} \int_{\mathcal{S}_{+}} \varphi\left(\alpha_{n_{j}}, s\right) d \mu(s) \\
& \leq \limsup _{j} \int_{\mathcal{S}_{+}} \varphi\left(\alpha_{n_{j}}, s\right) d \mu^{n_{j}}(s)+\epsilon^{\prime} \\
& \leq \limsup _{j} \int_{\mathcal{S}} \varphi\left(\alpha_{n_{j}}, s\right) d \mu^{n_{j}}(s)+\epsilon^{\prime}=\ell_{\varphi}^{*}(\mu)+\epsilon^{\prime}
\end{aligned}
$$

by using the limit (2). Since $\epsilon^{\prime}$ was arbitrary, we see that $\lim _{j} \ell_{\varphi}\left(\alpha_{n_{j}}, \mu\right)=$ $\ell_{\varphi}^{*}(\mu)$ as desired. 
Proof of Theorem 1 under Assumption C. Part (a) of the theorem follows immediately from Lemma 5, which implies that for any $\epsilon>0, H(\epsilon)>0$, whence we can apply Proposition 1.

For part (b), we focus on the case when $\left\{p_{q}\right\}$ is supported completely on a single $q$ and prove the result by contradiction. Assume that $\varphi$ is not structure consistent, so that there exists a $\mu \in \mathcal{M}(\mathcal{S})$ satisfying

$$
\ell_{\varphi}^{*}(\mu)=\inf _{\alpha} \ell_{\varphi}(\alpha, \mu)=\inf _{\alpha}\left\{\ell_{\varphi}(\alpha, \mu) \mid \alpha \notin \underset{\alpha^{\prime}}{\operatorname{argmin}} \ell\left(\alpha^{\prime}, \mu\right)\right\} .
$$

There exists a sequence $\alpha_{n} \notin \operatorname{argmin}_{\alpha} \ell(\alpha, \mu)$ with $\ell_{\varphi}\left(\alpha_{n}, \mu\right) \rightarrow \ell_{\varphi}^{*}(\mu)$. Define the sequence of functions $f_{n}$ with $f_{n}(q)=\alpha_{n}$, and let $\mu_{q}=\mu$. In this case, we have $R_{\varphi}(f)=\ell_{\varphi}(f(q), \mu)$, but by the finiteness of the range of $\ell(\cdot, \mu)$, there is some $\epsilon>0$ such that $\ell\left(\alpha_{n}, \mu\right) \geq \inf _{\alpha} \ell(\alpha, \mu)+\epsilon$ for all $n$. Thus we see that although $R_{\varphi}\left(f_{n}\right) \rightarrow R_{\varphi}^{*}$, we have

$$
R\left(f_{n}\right)=\ell\left(f_{n}(q), \mu\right)=\ell\left(\alpha_{n}, \mu\right) \not \rightarrow R^{*}=\inf _{\alpha} \ell(\alpha, \mu) .
$$

Thus we fail to have consistency as desired.

Proof of Theorem 1 under Assumption C' and coercivity. Now we turn to proving the result of Theorem 1 when Assumption C' holds, but we also assume that for any $\mu \in \mathcal{M}(\mathcal{S})$, the function $\alpha \mapsto \ell_{\varphi}(\alpha, \mu)$ is 0 -coercive. We begin by noting that since $\mathcal{S}$ is compact, Prohorov's Theorem implies the collection of probability measures defined on $\mathcal{S}$ is compact with respect to the topology of weak convergence [2, Chapter 5]. We let $\rightsquigarrow$ denote weak convergence (or convergence in distribution) of measures. To prove the result, all we require is an analogue of Lemma 5 .

Before proceeding, we recall a few results from variational analysis, referring to the book of Rockafellar and Wets [7] for a deeper exploration of these issues. For notational convenience, for a function $g: \mathbb{R}^{m} \rightarrow \mathbb{R}$ we define the approximate minimal set

$$
\epsilon-\operatorname{argmin} g:=\left\{x \in \mathbb{R}^{m}: g(x) \leq \inf _{x \in \operatorname{dom} g} g(x)+\epsilon\right\} .
$$

Proposition 4. Let $g_{n}: \mathbb{R}^{m} \rightarrow \mathbb{R}$ be a sequence of closed convex functions converging pointwise to a 0 -coercive function $\mathrm{g}$. Then $\mathrm{g}$ is closed convex. Moreover, if $\epsilon_{n}>0$ is a sequence with $\epsilon_{n} \rightarrow 0$, then any sequence $x_{n} \in \epsilon_{n}$-argmin $g_{n}$ has a cluster point and

$$
\limsup _{n} g\left(x_{n}\right) \leq \inf _{x} g(x) .
$$


Proof. This is a recapitulation of results due to Rockafellar and Wets [7] and Hiriart-Urruty and Lemaréchal [4]. We begin by noting that since the $g_{n}$ converge pointwise, the function $g$ is convex [4, Thoerem IV.3.1.5]. Pointwise convergence of convex $g_{n}$ implies convergence of their epigraphs in the sense of set convergence [7, Theorem 7.17], and hence $g$ is closed convex [7, Proposition 7.4(a)]. Since $g$ is coercive (by assumption), Theorem 7.33 of [7] implies that there exists a bounded set $C \subset \mathbb{R}^{m}$ such that $\epsilon_{n}$-argmin $g_{n} \subset C$ for all suitably large $n$. The result also states that for any $\epsilon>0$, eventually $\epsilon_{n}$-argmin $g_{n} \subset \epsilon$-argmin $g$, and the sequence of non-empty sets $\epsilon_{n}$-argmin $g_{n}$ has all its cluster points in $\operatorname{argmin} g$.

We now give our promised analogue of Lemma 5 .

LEMMA 6. Let $\varphi$ be structure consistent and assume that for each $\mu \in$ $\mathcal{M}(\mathcal{S})$, the function $\alpha \mapsto \ell_{\varphi}(\alpha, \mu)$ is 0-coercive. Then the suboptimality function (8) satisfies $H(\epsilon)>0$ for all $\epsilon>0$.

Proof. The proof follows that of Lemma 5, but we require the somewhat stronger tools of Proposition 4, since $\mathcal{S}$ is no longer necessarily finite.

Assume for the sake of contradiction that there exists $\epsilon>0$ and a sequence of pairs $\left(\alpha_{n}, \mu^{n}\right) \in \mathbb{R}^{m} \times \mathcal{M}(\mathcal{S})$ satisfying

$$
\ell\left(\alpha_{n}, \mu^{n}\right)-\inf _{\alpha^{\prime}} \ell\left(\alpha^{\prime}, \mu^{n}\right) \geq \epsilon \quad \text { and } \quad \ell_{\varphi}\left(\alpha_{n}, \mu^{n}\right)-\ell_{\varphi}^{*}\left(\mu^{n}\right) \rightarrow 0 .
$$

Since $\mathcal{M}(\mathcal{S})$ is compact with respect to weak convergence (Prohorov's theorem), we can take a subsequence $n_{j}$ such that $\mu^{n_{j}} \rightsquigarrow \mu$ for some $\mu \in \mathcal{M}(\mathcal{S})$.

By weak-convergence, we have the pointwise convergence

$$
\lim _{j} \ell_{\varphi}\left(\alpha, \mu^{n_{j}}\right)=\ell_{\varphi}(\alpha, \mu) \text { for all } \alpha \in \mathbb{R}^{m} .
$$

Of course, the funtions $\ell_{\varphi}\left(\cdot, \mu^{n}\right)$ and $\ell_{\varphi}(\cdot, \mu)$ are convex, and the latter is 0-coercive, whence Proposition 4 applies. We find in particular

$$
\limsup _{j} \ell_{\varphi}\left(\alpha_{n_{j}}, \mu\right) \leq \inf _{\alpha} \ell_{\varphi}(\alpha, \mu)=\ell_{\varphi}^{*}(\mu) .
$$

Lemma 4 holds under Assumption C', and the proof is, mutatis mutandis, the same. Thus, Applying Lemma 4, we find that $\alpha_{n_{j}} \in \operatorname{argmin}_{\alpha} \ell(\alpha, \mu)$ eventually, whence $\ell\left(\alpha_{n_{j}}, \mu\right)-\inf _{\alpha^{\prime}} \ell\left(\alpha^{\prime}, \mu\right)=0$. Since $\mu \mapsto \ell(\alpha, \mu)$ is continuous, this contradicts the assumption that $\ell\left(\alpha_{n}, \mu^{n}\right)-\inf _{\alpha^{\prime}} \ell\left(\alpha^{\prime}, \mu^{n}\right) \geq \epsilon$ for all $n$, which is our desired contradiction. So $H(\epsilon)>0$. 
Finalizing the proof. The remainder of the proof is completely identical to that under Assumption C, but now instead of Lemma 5 we apply Lemma 6 .

8.3. Proof of Lemma 1. Consider the function

$$
g(p)=\sum_{i=1}^{m} \frac{1}{F(i)} p_{i} \prod_{j=1}^{i-1}\left(1-p_{j}\right)
$$

and assume that $p_{i}<p_{i+1}$ for some index $i$. Let $p^{\prime}=p$ except that the values of $p_{i}$ and $p_{i+1}$ are swapped. Then

$$
\begin{aligned}
g(p)-g\left(p^{\prime}\right)= & \frac{1}{F(i)} p_{i} \prod_{j=1}^{i-1}\left(1-p_{j}\right)+\frac{1}{F(i+1)} p_{i+1} \prod_{j=1}^{i}\left(1-p_{j}\right) \\
& \quad-\frac{1}{F(i)} p_{i+1} \prod_{j=1}^{i-1}\left(1-p_{j}\right)-\frac{1}{F(i+1)} p_{i}\left(1-p_{i+1}\right) \prod_{j=1}^{i-1}\left(1-p_{i}\right) \\
= & \frac{1}{F(i)}\left(p_{i}-p_{i+1}\right) \prod_{j=1}^{i-1}\left(1-p_{j}\right) \\
& \quad+\frac{1}{F(i+1)}\left(p_{i+1}\left(1-p_{i}\right)-p_{i}\left(1-p_{i+1}\right)\right) \prod_{j=1}^{i-1}\left(1-p_{j}\right) \\
= & \left(p_{i}-p_{i+1}\right)\left[\frac{1}{F(i)}-\frac{1}{F(i+1)}\right] \prod_{j=1}^{i-1}\left(1-p_{j}\right)<0
\end{aligned}
$$

since $F$ is an increasing function. Thus swapping $p_{i}$ and $p_{i+1}$ increases the objective $g(p)$, proving the lemma.

9. Proofs of Uniform Laws. In this section, we provide the proof of Theorem 4. The proof has two parts, the first controlling the expectation (20), the second controlling the convergence of the empirical risk $\widehat{R}_{\varphi, n}$ to its expectation $R_{\varphi, n}$.

9.1. Expectation. We begin by studying the expectation (20). Our goal is to understand the rate at which we have convergence

$$
R_{\varphi, n}(f) \rightarrow \sum_{q} p_{q} \mathbb{E}_{q}\left[\varphi\left(f(q), s\left(Y_{1}, \ldots, Y_{k}\right) \mid Q=q\right)\right]
$$

If the convergence (3) occurs sufficiently quickly, then we can allow $k$ to increase to infinity, capturing the asymptotic surrogate risk (6). The following 
lemma, whose proof we provide in Appendix 9.3, gives sufficient conditions for such a convergence result to hold.

Lemma 7. Let Assumptions $D$ and $E$ hold for the sequence of function classes $\mathcal{F}_{n}$. There exists a constant $C\left(K_{1}, \beta\right)<\infty$, such that

$$
\begin{gathered}
\sup _{f \in \mathcal{F}_{n}}\left|R_{\varphi, n}(f)-\sum_{q} p_{q} \mathbb{E}\left[\varphi\left(f(Q), s\left(Y_{1}, \ldots, Y_{k}\right)\right) \mid Q=q\right]\right| \\
\leq C\left(K_{1}, \beta\right) B_{n} n^{-\frac{\beta}{1+\beta}}(k+\log k+2 \log n)^{\frac{\beta}{1+\beta}} .
\end{gathered}
$$

Lemma 7 is suggestive of Condition II and its requirements on $B_{n}$ and $k_{n}$. We now show how Conditions I or I' and Condition II imply that $\sup _{f \in \mathcal{F}_{n}}\left|R_{\varphi, n}(f)-R_{\varphi}(f)\right| \rightarrow 0$. First, we show how Condition I implies I'. Indeed, since $\sum_{q} p_{q}=1$, Condition I implies that for any $f \in \mathcal{F}_{n}$

$$
\begin{aligned}
& \left|R_{\varphi}(f)-\sum_{q} p_{q} \mathbb{E}_{q}\left[\varphi\left(f(q), s\left(Y_{1: k_{n}}\right)\right)\right]\right| \\
& \leq \sum_{q} p_{q}\left|\mathbb{E}_{q}\left[\varphi\left(f(q), s\left(Y_{1: k_{n}}\right)\right)\right]-\lim _{k^{\prime}} \mathbb{E}_{q}\left[\varphi\left(f(q), s\left(Y_{1: k^{\prime}}\right)\right)\right]\right| \leq C B_{n} k_{n}^{-\rho},
\end{aligned}
$$

which tends to 0 whenever $B_{n}=o\left(k_{n}^{\rho}\right)$. Then for any $\epsilon_{n}>0$ we obtain

$$
\max _{i \in\left[N\left(\epsilon_{n}, n\right)\right]} \inf _{f \in \mathcal{F}_{n}^{i}}\left|R_{\varphi}(f)-\sum_{q} p_{q} \mathbb{E}_{q}\left[\varphi\left(f(q), s\left(Y_{1: k_{n}}\right)\right)\right]\right| \leq C B_{n} k_{n}^{-\rho} \rightarrow 0,
$$

and we may assume $\epsilon_{n} L_{n} \rightarrow 0$ in Condition I'. This established, the following lemma captures the convergence of expectations (see Appendix 9.4).

Lemma 8. Let Condition I' and $k_{n} B_{n}^{(1+\beta) / \beta}=o(n)$ hold. Under Assumptions $D$ and $E$, as $n \rightarrow \infty$

$$
\sup _{f \in \mathcal{F}_{n}}\left|R_{\varphi, n}(f)-R_{\varphi}(f)\right| \rightarrow 0 .
$$

9.2. High probability guarantees. We now turn to demonstrating highprobability convergence of the empirical risk (19) to its expectation. Our approach makes use of the smoothness Assumption E along with a covering number argument [e.g., 10]. We use the bounded differences inequality [6] to demonstrate the convergence of the empirical risk (19) to its expectation. We begin by viewing $\widehat{R}_{\varphi, n}(f)$ as a random function of the $n$ query preference evaluation pairs $\left(Q_{1}, Y_{1}\right), \ldots,\left(Q_{n}, Y_{n}\right)$; define

$$
F\left(\left(Q_{1}, Y_{1}\right), \ldots,\left(Q_{n}, Y_{n}\right)\right):=\widehat{R}_{\varphi, n}(f) .
$$


We have the following lemma, proved in Appendix 9.5.

Lemma 9. Assume $\left|\varphi\left(f(q), s\left(Y_{1}, \ldots, Y_{m}\right)\right)\right| \leq B$ for all $f \in \mathcal{F}$. Then

$\left|F\left(\left(Q_{1}, Y_{1}\right), \ldots,\left(Q_{n}, Y_{n}\right)\right)-F\left(\left(Q_{1}, Y_{1}\right), \ldots,\left(Q_{j}^{\prime}, Y_{j}^{\prime}\right), \ldots,\left(Q_{n}, Y_{n}\right)\right)\right| \leq \frac{4 k B}{n}$ for all $j$ and $\left(Q_{j}^{\prime}, Y_{j}^{\prime}\right)$.

As a consequence of Lemma 9, the bounded differences inequality [6] implies that since $\left|\varphi\left(f(q), s\left(Y_{1}, \ldots, Y_{k}\right)\right)\right| \leq B_{n}$ for any fixed $f \in \mathcal{F}_{n}$,

$$
\mathbb{P}\left(\left|\widehat{R}_{\varphi, n}(f)-R_{\varphi, n}(f)\right| \geq \epsilon\right) \leq 2 \exp \left(-\frac{n \epsilon^{2}}{8 k^{2} B_{n}^{2}}\right) .
$$

Using Assumption E, we can now apply our covering number argument to get a uniform bound on the deviations $\widehat{R}_{\varphi, n}(f)-R_{\varphi, n}(f)$. We obtain

Lemma 10. Let Assumptions $E$ and $F$ and Condition II hold. Then

$$
\sup _{f \in \mathcal{F}_{n}}\left|\widehat{R}_{\varphi, n}(f)-R_{\varphi, n}(f)\right| \stackrel{p}{\rightarrow} 0 \text {. }
$$

See Appendix 9.6 for the proof of the lemma, noting that the bound (4) guarantees almost sure and expected convergence in the conclusion of the lemma under the conditions in the remark following Theorem 4.

To complete the proof of Theorem 4, note that Lemma 8 guarantees the uniform convergence of the difference of expectations $R_{\varphi, n}(f)-R_{\varphi}(f)$; applying the triangle inequality in Lemma 10 completes the argument.

9.3. Proof of Lemma \%. We begin by fixing some $q_{0}$ and splitting the summation (20) into two terms:

$$
\begin{aligned}
R_{\varphi, n}(f)= & \sum_{q \leq q_{0}}\left[\sum_{m=1}^{n} m \mathbb{P}_{n}\left(\widehat{n}_{q}=m\right) \mathbb{E}\left[\varphi\left(f(Q), s\left(Y_{1}, \ldots, Y_{m \wedge k}\right)\right) \mid Q=q\right]\right] \\
& +\sum_{q>q_{0}}\left[\sum_{m=1}^{n} m \mathbb{P}_{n}\left(\widehat{n}_{q}=m\right) \mathbb{E}\left[\varphi\left(f(Q), s\left(Y_{1}, \ldots, Y_{m \wedge k}\right)\right) \mid Q=q\right]\right],
\end{aligned}
$$

the sum over queries $q \leq q_{0}$ and the sum over $q>q_{0}$. We control each sum in turn, using a Chernoff bound to control the first and the fact that $\sum_{q>q_{0}} p_{q} \rightarrow 0$ quickly enough as $q_{0} \uparrow \infty$ (due to the power law assumption D) for the second. We also use the shorthand notation $\mathbb{E}_{q}\left[\varphi\left(f(q), s\left(Y_{1: k}\right)\right)\right]$ for the quantity $\mathbb{E}\left[\varphi\left(f(Q), s\left(Y_{1}, \ldots, Y_{k}\right)\right) \mid Q=q\right]$ to keep the arguments neater.

We begin by studying the expectation of the more probable queries, for which we require a version of the Chernoff bound: 
Lemma 11 (Multiplicative Chernoff bound). Let $X_{i}$ be i.i.d. Bernoulli random variables with $\mathbb{P}\left(X_{i}=1\right)=p$. For any $\delta \geq 0$,

$$
\mathbb{P}\left(\sum_{i=1}^{n} X_{i}<(1-\delta) n p\right) \leq \exp \left(-\frac{n p \delta^{2}}{2}\right) .
$$

As an immediate consequence of the Chernoff bound, we see that for any query $q$ and empirical count $\widehat{n}_{q}$, we have

$$
\begin{aligned}
\mathbb{P}_{n}\left(\widehat{n}_{q}<k\right) & =\mathbb{P}_{n}\left(\widehat{n}_{q}<\frac{k}{n p_{q}} n p_{q}\right)=\mathbb{P}_{n}\left[\widehat{n}_{q}<\left(1-\left(1-\frac{k}{n p_{q}}\right)\right) n p_{q}\right] \\
& \leq \exp \left(-\frac{n p_{q}}{2}\left(1-\frac{k}{n p_{q}}\right)^{2}\right)=\exp \left(-\frac{n p_{q}}{2}+k-\frac{k^{2}}{2 n p_{q}}\right) .
\end{aligned}
$$

For any $\epsilon \in(0,1)$, to have $\mathbb{E}\left[\widehat{n}_{q} 1\left(\widehat{n}_{q}<k\right)\right] \leq \epsilon n p_{q}$, it is sufficient that

$$
n>\frac{2}{p_{q}}\left[k+\log k+\log \frac{1}{\epsilon}\right] \text {. }
$$

Indeed, for such $n$ we have $n p_{q} \geq 1$ and

$$
\begin{aligned}
\mathbb{E}\left[\widehat{n}_{q} 1\left(\widehat{n}_{q}<k\right)\right]=\sum_{m=1}^{k-1} m \mathbb{P}_{n}\left(\widehat{n}_{q}=m\right) & \leq k \mathbb{P}_{n}\left(\widehat{n}_{q}<k\right) \leq k \exp \left(-\frac{n p_{q}}{2}+k\right) \\
& \leq k \exp (-\log k+\log \epsilon)=\epsilon \leq \epsilon n p_{q}
\end{aligned}
$$

For fixed $\epsilon>0$ and $n \in \mathbb{N}$, we let $q_{0}=q_{0}(\epsilon, n)$ for

$$
q_{0}(\epsilon, n):=\max \left\{q: \frac{2}{p_{q}}\left[k+\log k+\log \frac{1}{\epsilon}\right]<n\right\} .
$$

Then for all $q \leq q_{0}$, since $\sum_{m=1}^{n} m \mathbb{P}_{n}\left(\widehat{n}_{q}=m\right)=n p_{q}$ and $k \mathbb{P}_{n}\left(\widehat{n}_{q}<k\right) \leq \epsilon$, we immediately see that

$$
\begin{aligned}
& (1-\epsilon) n p_{q} \mathbb{E}_{q}\left[\varphi\left(f(q), s\left(Y_{1}, \ldots, Y_{k}\right)\right)\right] \\
& \leq \sum_{m=k}^{n} m \mathbb{P}_{n}\left(\widehat{n}_{q}=m\right) \mathbb{E}_{q}\left[\varphi\left(f(q), s\left(Y_{1}, \ldots, Y_{k}\right)\right)\right] \\
& \leq \sum_{m=1}^{n} m \mathbb{P}_{n}\left(\widehat{n}_{q}=m\right) \mathbb{E}_{q}\left[\varphi\left(f(q), s\left(Y_{1}, \ldots, Y_{m \wedge k}\right)\right]\right.
\end{aligned}
$$


and additionally that

$$
\begin{aligned}
\sum_{m=1}^{k-1} m \mathbb{P}\left(\widehat{n}_{q}=m\right) \mathbb{E}_{q}\left[\varphi\left(f(q), s\left(Y_{1: m}\right)\right)\right] & +\sum_{m=k}^{n} m \mathbb{P}\left(\widehat{n}_{q}=m\right) \mathbb{E}_{q}\left[\varphi\left(f(q), s\left(Y_{1: k}\right)\right)\right] \\
& \leq \epsilon B_{n}+n p_{q} \mathbb{E}_{q}\left[\varphi\left(f(q), s\left(Y_{1}, \ldots, Y_{k}\right)\right)\right]
\end{aligned}
$$

by applying Assumption $\mathrm{E}$ on the boundedness of $\varphi$. We thus see that

$$
\begin{aligned}
& (1-\epsilon) \sum_{q \leq q_{0}} p_{q} \mathbb{E}_{q}\left[\varphi\left(f(q), s\left(Y_{1: k}\right)\right)\right] \leq R_{\varphi, n}(f) \\
& \quad \leq \sum_{q \leq q_{0}} p_{q} \mathbb{E}_{q}\left[\varphi\left(f(q), s\left(Y_{1: k}\right)\right)\right]+\frac{q_{0}(\epsilon, n) \epsilon B_{n}}{n}+B_{n} \sum_{q>q_{0}} p_{q}
\end{aligned}
$$

for all $f \in \mathcal{F}_{n}$. The sandwich inequality (7) suggests that we should have our desired convergence statement (3) so long as the bound $B_{n}$ does not grow too quickly.

Now we use Assumption D to complete the proof by bounding $q_{0}$ and $\sum_{q>q_{0}} p_{q}$. Recalling the definition (6) of $q_{0}$, we note that for $q>q_{0}$,

$$
p_{q} \leq \min \left\{K_{1} q^{-\beta-1}, \frac{2\left(k+\log k+\log \epsilon^{-1}\right)}{n}\right\} \text {. }
$$

Let

$$
q_{0}^{*}:=\left(\frac{K_{1} n}{2\left(k+\log k+\log \epsilon^{-1}\right)}\right)^{\frac{1}{\beta+1}}
$$

denote the $q$ solving $K_{1} q^{-\beta-1}=2\left(k+\log k+\log \epsilon^{-1}\right) / n$. Then

$$
\begin{aligned}
\sum_{q>q_{0}} p_{q} & \leq \int_{1}^{q_{0}^{*}} \frac{2\left(k+\log k+\log \epsilon^{-1}\right)}{n} d q+\int_{q_{0}^{*}}^{\infty} K_{1} q^{-\beta-1} d q \\
& \leq 2 q_{0}^{*} \frac{k+\log k+\log \frac{1}{\epsilon}}{n}+\frac{K_{1}}{\beta} q_{0}^{*-\beta} \leq C\left(\frac{k+\log k+\log \frac{1}{\epsilon}}{n}\right)^{\frac{\beta}{1+\beta}}
\end{aligned}
$$

where $C$ is a constant dependent on $K_{1}$ and $\beta$. Lastly, we bound $q_{0}(\epsilon, n)$. Since $p_{q} \leq K_{1} q^{-\beta-1}$, the inequality (5) can be satisfied only if

$$
q \leq\left(\frac{K_{1} n}{2\left(k+\log k+\log \epsilon^{-1}\right)}\right)^{\frac{1}{1+\beta}}
$$

Choosing $\epsilon$ to be a function of $n$ via $\epsilon=1 / n^{2}$, we can use Assumption $\mathrm{E}$ and the sandwich inequality (7) to find that there is constant $C^{\prime}$ - dependent 
on $K_{1}$ and $\beta$-such that

$$
\begin{aligned}
& \sum_{q} p_{q} \mathbb{E}_{q}\left[\varphi\left(f(q), s\left(Y_{1: k}\right)\right)\right]-C^{\prime} B_{n}\left(n^{-2}+\left(\frac{k+\log k+2 \log n}{n}\right)^{\frac{\beta}{1+\beta}}\right) \\
& \leq R_{\varphi, n}(f) \\
& \leq \sum_{q} p_{q} \mathbb{E}_{q}\left[\varphi\left(f(q), s\left(Y_{1: k}\right)\right)\right]+C^{\prime} B_{n} n^{\frac{-2-3 \beta}{1+\beta}}(k+\log k+2 \log n)^{-\frac{1}{1+\beta}} \\
& \quad+C^{\prime} B_{n} n^{\frac{-\beta}{1+\beta}}(k+\log k+2 \log n)^{\frac{\beta}{1+\beta}}
\end{aligned}
$$

The two-sided bound (8) implies the lemma.

9.4. Proof of Lemma 8. Fix $\epsilon>0$ and let $f_{n}^{i} \in \mathcal{F}_{n}^{i}$ be an arbitrary member of each of the sets $\mathcal{F}_{n}^{i}$. Then

$$
\begin{aligned}
& \sup _{f \in \mathcal{F}_{n}}\left|R_{\varphi}(f)-\sum_{q} p_{q} \mathbb{E}_{q}\left[\varphi\left(f(q), s\left(Y_{1: k_{n}}\right)\right)\right]\right| \\
& =\max _{i \in[N(\epsilon, n)]} \sup _{f \in \mathcal{F}_{n}^{i}}\left|\sum_{q} p_{q} \ell_{\varphi}\left(f(q), \mu_{q}\right)-\sum_{q} p_{q} \mathbb{E}_{q}\left[\varphi\left(f(q), s\left(Y_{1: k_{n}}\right)\right)\right]\right| \\
& \leq \max _{i \in[N(\epsilon, n)]}\left|R_{\varphi}\left(f_{n}^{i}\right)-\sum_{q} p_{q} \mathbb{E}_{q}\left[\varphi\left(f_{n}^{i}(q), s\left(Y_{1: k_{n}}\right)\right)\right]\right| \\
& \quad+\max _{i \in[N(\epsilon, n)]} \sup _{f \in \mathcal{F}_{n}^{i}}\left|\sum_{q} p_{q}\left[\ell_{\varphi}\left(f_{n}^{i}(q), \mu_{q}\right)-\ell_{\varphi}\left(f(q), \mu_{q}\right)\right]\right| \\
& \quad+\max _{i \in[N(\epsilon, n)]} \sup _{f \in \mathcal{F}_{n}^{i}}\left|\sum_{q} p_{q}\left(\mathbb{E}_{q}\left[\varphi\left(f(q), s\left(Y_{1: k_{n}}\right)\right)\right]-\mathbb{E}_{q}\left[\varphi\left(f_{n}^{i}(q), s\left(Y_{1: k_{n}}\right)\right)\right]\right)\right|
\end{aligned}
$$

by the triangle inequality. Applying Assumption $\mathrm{E}$ and that $\mathcal{F}_{n}^{i}$ has radius $\epsilon$, the final two terms are each bounded by $L_{n} \epsilon$, which implies the bound

$$
\max _{i \in[N(\epsilon, n)]}\left|R_{\varphi}\left(f_{n}^{i}\right)-\sum_{q} p_{q} \mathbb{E}_{q}\left[\varphi\left(f_{n}^{i}(q), s\left(Y_{1: k_{n}}\right)\right)\right]\right|+2 L_{n} \epsilon .
$$

Noting that $f_{n}^{i}$ was arbitrary, we can strengthen the bound (9) to

$$
\begin{aligned}
& \sup _{f \in \mathcal{F}_{n}}\left|R_{\varphi}(f)-\sum_{q} p_{q} \mathbb{E}_{q}\left[\varphi\left(f(q), s\left(Y_{1: k_{n}}\right)\right)\right]\right| \\
& \quad \leq \max _{i \in[N(\epsilon, n)]} \inf _{f \in \mathcal{F}_{n}^{i}}\left|R_{\varphi}(f)-\sum_{q} p_{q} \mathbb{E}_{q}\left[\varphi\left(f(q), s\left(Y_{1: k_{n}}\right)\right)\right]\right|+2 L_{n} \epsilon .
\end{aligned}
$$


Applying Lemma 7 completes the proof.

9.5. Proof of Lemma 9. Without loss of generality, let $j=1$, and fix the query judgment pairs $\left(Q_{1}, Y_{1}\right)=(q, Y)$ and $\left(Q_{1}^{\prime}, Y_{1}^{\prime}\right)=\left(q^{\prime}, Y^{\prime}\right)$ with $Q_{i}^{\prime}:=Q_{i}$ for all $i \in\{2, \ldots, n\}$. Recall the notation $\mathcal{B}(q)$ from Section 4 , and for any $r \in \mathcal{Q}$, let $\mathcal{B}^{\prime}(r):=\left\{i \in[n] \mid Q_{i}^{\prime}=r\right\}$ and define $\widehat{n}_{r}^{\prime}=\left|\mathcal{B}^{\prime}(r)\right|$. In addition, let $Y_{i_{1}: i_{k}}$ denote $Y_{i_{1}}, \ldots, Y_{i_{k}}$.

We focus first on the case in which $q \neq q^{\prime}$. Note that by definition (19)

$n F\left((q, Y),\left(Q_{2}, Y_{2}\right), \ldots,\left(Q_{n}, Y_{n}\right)\right)-n F\left(\left(q^{\prime}, Y^{\prime}\right),\left(Q_{2}, Y_{2}\right), \ldots,\left(Q_{n}, Y_{n}\right)\right)$

$$
\begin{aligned}
& =\widehat{n}_{q}\left(\begin{array}{c}
\widehat{n}_{q} \\
k
\end{array}\right)^{-1} \sum_{\substack{i_{1}<\cdots<i_{k}, i_{j} \in \mathcal{B}(q)}} \varphi\left(f(q), s\left(Y_{i_{1}: i_{k}}\right)\right)-\left(\widehat{n}_{q}-1\right)\left(\begin{array}{c}
\widehat{n}_{q}-1 \\
k
\end{array}\right)^{-1} \sum_{\substack{i_{1}<\cdots<i_{k}, i_{j} \in \mathcal{B}^{\prime}(q)}} \varphi\left(f(q), s\left(Y_{i_{1}: i_{k}}\right)\right) \\
& (10 \mathrm{~b}) \\
& +\widehat{n}_{q^{\prime}}\left(\begin{array}{c}
\widehat{n}_{q^{\prime}} \\
k
\end{array}\right)^{-1} \sum_{\substack{i_{1}<\cdots<i_{k}, i_{j} \in \mathcal{B}\left(q^{\prime}\right)}} \varphi\left(f\left(q^{\prime}\right), s\left(Y_{i_{1}: i_{k}}\right)\right)-\left(\widehat{n}_{q^{\prime}}+1\right)\left(\begin{array}{c}
\widehat{n}_{q^{\prime}}+1 \\
k
\end{array}\right)^{-1} \sum_{\substack{i_{1}<\cdots<i_{k}, i_{j} \in \mathcal{B}^{\prime}\left(q^{\prime}\right)}} \varphi\left(f\left(q^{\prime}\right), s\left(Y_{i_{1}: i_{k}}^{\prime}\right)\right) .
\end{aligned}
$$

We first bound the term (10a). When $\widehat{n}_{q} \leq k$, this term becomes

$$
\widehat{n}_{q} \varphi\left(f(q), s\left(Y_{i_{1}: i_{\widehat{n}_{q}}}\right)\right)-\left(\widehat{n}_{q}-1\right) \varphi\left(f(q), s\left(Y_{i_{2}: i_{\widehat{n}_{q}}}\right)\right)
$$

where $i_{1}, \ldots, i_{\widehat{n}_{q}}$ denote the distinct elements of $\mathcal{B}(q)$. By assumption, each of the terms $\varphi$ is bounded by $B$, so this difference is at most $2 \widehat{n}_{q} B \leq 2 k B$.

When $\widehat{n}_{q}>k$, we note that the term (10a) equals

$$
\begin{gathered}
{\left[\widehat{n}_{q}\left(\begin{array}{c}
\widehat{n}_{q} \\
k
\end{array}\right)^{-1}-\left(\widehat{n}_{q}-1\right)\left(\begin{array}{c}
\widehat{n}_{q}-1 \\
k
\end{array}\right)^{-1}\right] \sum_{\substack{i_{1}<\cdots<i_{k}, i_{j} \in \mathcal{B}^{\prime}(q)}} \varphi\left(f(q), s\left(Y_{i_{1}: i_{k}}\right)\right)} \\
+\widehat{n}_{q}\left(\begin{array}{c}
\widehat{n}_{q} \\
k
\end{array}\right)^{-1} \sum_{\substack{i_{2}<\ldots<i_{k}, i_{j} \in \mathcal{B}^{\prime}(q)}} \varphi\left(f(q), s\left(Y, Y_{i_{2}: i_{k}}\right)\right) .
\end{gathered}
$$

Since there are $\left(\begin{array}{c}\widehat{n}_{q}-1 \\ k\end{array}\right)$ terms in the summation (11a) and $\left(\begin{array}{c}\widehat{n}_{q}-1 \\ k-1\end{array}\right)$ terms in the summation (11b), and for any $m, k \in \mathbb{N}$ with $m>k$,

$$
\begin{aligned}
m\left(\begin{array}{c}
m \\
k
\end{array}\right)^{-1}-(m-1)\left(\begin{array}{c}
m-1 \\
k
\end{array}\right)^{-1} & =\frac{k !(m-k) !}{(m-1) !}-(m-1) \frac{(m-1-k) ! k !}{(m-1) !} \\
& =(1-k)\left(\begin{array}{c}
k \\
m-1
\end{array}\right)^{-1},
\end{aligned}
$$


we use the fact that $|\varphi| \leq B$ to conclude that

$$
\begin{aligned}
\mid \widehat{n}_{q}\left(\begin{array}{c}
\widehat{n}_{q} \\
k
\end{array}\right)^{-1} \sum_{\substack{i_{1}<\cdots<i_{k}, i_{j} \in \mathcal{B}(q)}} \varphi\left(f(q), s\left(Y_{i_{1}: i_{k}}\right)\right) \\
\quad-\left(\widehat{n}_{q}-1\right)\left(\begin{array}{c}
\widehat{n}_{q}-1 \\
k
\end{array}\right)^{-1} \sum_{\substack{i_{1}<\cdots<i_{k}, i_{j} \in \mathcal{B}^{\prime}(q)}} \varphi\left(f(q), s\left(Y_{i_{1}: i_{k}}\right)\right) \mid \\
\leq|k-1| B+\widehat{n}_{q}\left(\begin{array}{c}
\widehat{n}_{q} \\
k
\end{array}\right)^{-1}\left(\begin{array}{c}
\widehat{n}_{q}-1 \\
k-1
\end{array}\right) B=|k-1| B+\widehat{n}_{q} \frac{k}{\widehat{n}_{q}} B \leq 2 k B .
\end{aligned}
$$

Hence, the term (10a) can be bounded by $2 k B$ for any choice of $\widehat{n}_{q}$. Bounding the term $(10 \mathrm{~b})$ requires an entirely similar combinatorial argument and again yields the bound $2 k B$ for a total bound of $4 k B$.

All that remains is to control the difference of the functions $F$ when $q=q^{\prime}$. When $\widehat{n}_{q} \leq k$, this difference is given by

$$
\left(\widehat{n}_{q} / n\right) \mid \varphi\left(f(q), s\left(Y_{i_{1}: i_{\widehat{n}_{q}}}\right)\right)-\varphi\left(f(q), s\left(Y_{i_{1}: i_{\widehat{n}_{q}}}^{\prime}\right) \mid \leq 2 k B / n .\right.
$$

When $\widehat{n}_{q} \geq k$, we have

$$
\begin{aligned}
& n F\left((q, Y),\left(Q_{2}, Y_{2}\right), \ldots,\left(Q_{n}, Y_{n}\right)\right)-n F\left(\left(q, Y^{\prime}\right),\left(Q_{2}, Y_{2}\right), \ldots,\left(Q_{n}, Y_{n}\right)\right) \\
& =\widehat{n}_{q}\left(\begin{array}{c}
\widehat{n}_{q} \\
k
\end{array}\right)^{-1} \sum_{\substack{i_{1}<\cdots<i_{k}, i_{j} \in \mathcal{B}(q)}}\left[\varphi\left(f(q), s\left(Y_{i_{1}: i_{k}}\right)\right)-\varphi\left(f(q), s\left(Y_{i_{1}: i_{k}}^{\prime}\right)\right)\right] \\
& =\widehat{n}_{q}\left(\begin{array}{c}
\widehat{n}_{q} \\
k
\end{array}\right)^{-1} \sum_{\substack{1=i_{1}<\cdots<i_{k} \\
i_{j} \in \mathcal{B}(q)}}\left[\varphi\left(f(q), s\left(Y, Y_{i_{2}: i_{k}}\right)\right)-\varphi\left(f(q), s\left(Y^{\prime}, Y_{i_{2}: i_{k}}\right)\right)\right] .
\end{aligned}
$$

There are $\left(\begin{array}{c}\widehat{n}_{q}-1 \\ k-1\end{array}\right)$ terms in the final summation, and since

$$
m\left(\begin{array}{c}
m \\
k
\end{array}\right)^{-1}\left(\begin{array}{c}
m-1 \\
k-1
\end{array}\right)=m \frac{k !(m-k) !}{m !} \cdot \frac{(m-1) !}{(k-1) !(m-k) !}=k,
$$

the difference (12) is bounded by $2 k B$.

9.6. Proof of Lemma 10. Let $\epsilon^{\prime}>0$ be arbitrary. Use Assumption $\mathrm{F}$ and partition $\mathcal{F}_{n}$ into $N=N\left(\epsilon^{\prime}, n\right)<\infty$ subsets $\mathcal{F}_{n}^{1}, \ldots, \mathcal{F}_{n}^{N}$, and fix $f^{i} \in \mathcal{F}_{n}^{i}$ so that for any $f \in \mathcal{F}_{n}^{i}$ we have $\left\|f-f^{i}\right\| \leq \epsilon^{\prime}$. Then for any index $i \in$ 
$\left\{1, \ldots, N\left(\epsilon^{\prime}, n\right)\right\}$, we see that

$$
\begin{aligned}
& \sup _{f \in \mathcal{F}_{n}^{i}}\left|\widehat{R}_{\varphi, n}(f)-R_{\varphi, n}(f)\right| \\
& =\sup _{f \in \mathcal{F}_{n}^{i}}\left|\widehat{R}_{\varphi, n}(f)-\widehat{R}_{\varphi, n}\left(f^{i}\right)+\widehat{R}_{\varphi, n}\left(f^{i}\right)-R_{\varphi, n}\left(f^{i}\right)+R_{\varphi, n}\left(f^{i}\right)-R_{\varphi, n}(f)\right| \\
& \leq\left|\widehat{R}_{\varphi, n}\left(f^{i}\right)-R_{\varphi, n}\left(f^{i}\right)\right|+\sup _{f \in \mathcal{F}_{n}^{i}}\left|\widehat{R}_{\varphi, n}(f)-\widehat{R}_{\varphi, n}\left(f^{i}\right)\right|+\sup _{f \in \mathcal{F}_{n}^{i}}\left|R_{\varphi, n}(f)-R_{\varphi, n}\left(f^{i}\right)\right| .
\end{aligned}
$$

Assumption E guarantees that $\left|\widehat{R}_{\varphi, n}(f)-\widehat{R}_{\varphi, n}\left(f^{i}\right)\right| \leq L_{n}\left\|f-f^{i}\right\|$, so

$$
\sup _{f \in \mathcal{F}_{n}^{i}}\left|\widehat{R}_{\varphi, n}(f)-\widehat{R}_{\varphi, n}\left(f^{i}\right)\right| \leq L_{n} \epsilon^{\prime}
$$

The same argument applies to the difference $R_{\varphi, n}(f)-R_{\varphi, n}\left(f^{i}\right)$. Thus we use the fact that the classes $\mathcal{F}_{n}^{i}$ partition $\mathcal{F}_{n}$, that is, $\cup_{i=1}^{N} \mathcal{F}_{n}^{i} \supseteq \mathcal{F}_{n}$, and apply a union bound and the triangle inequality to find that

$$
\begin{aligned}
& \mathbb{P}\left(\sup _{f \in \mathcal{F}_{n}}\left|\widehat{R}_{\varphi, n}(f)-R_{\varphi, n}(f)\right| \geq \epsilon\right) \\
& =\mathbb{P}\left(\max _{i \in\left\{1, \ldots, N\left(\epsilon^{\prime}, n\right)\right\}} \sup _{f \in \mathcal{F}_{n}^{i}}\left|\widehat{R}_{\varphi, n}(f)-R_{\varphi, n}(f)\right| \geq \epsilon\right) \\
& \leq \sum_{i=1}^{N\left(\epsilon^{\prime}, n\right)} \mathbb{P}\left(\sup _{f \in \mathcal{F}_{n}^{i}}\left|\widehat{R}_{\varphi, n}(f)-R_{\varphi, n}(f)\right| \geq \epsilon\right) \\
& \leq \sum_{i=1}^{N\left(\epsilon^{\prime}, n\right)} \mathbb{P}\left(\left|\widehat{R}_{\varphi, n}\left(f^{i}\right)-R_{\varphi, n}\left(f^{i}\right)\right| \geq \epsilon-2 L_{n} \epsilon^{\prime}\right) .
\end{aligned}
$$

Choosing $\epsilon^{\prime}=\epsilon /\left(4 L_{n}\right)$, we can apply the concentration inequality (4) to conclude that

$$
\mathbb{P}\left(\sup _{f \in \mathcal{F}_{n}}\left|\widehat{R}_{\varphi, n}(f)-R_{\varphi, n}(f)\right| \geq \epsilon\right) \leq 2 \exp \left[\log N\left(\frac{\epsilon}{4 L_{n}}, n\right)-\frac{n \epsilon^{2}}{32 k^{2} B_{n}^{2}}\right] .
$$

This implies the statement of the lemma.

10. Proofs of Inconsistency Results. The proofs in this section are essentially present in the preliminary version [3] of this work. We present and extend them here for the convenience of the reader. 
10.1. Proof of Proposition 3. This proposition is a consequence of the fact that the feedback arc set problem [5] is $N P$-complete. In the feedback arc set problem, we are given a directed graph $G=(V, E)$ and an integer $k$ and need to determine whether there is a subset $E^{\prime} \subseteq E$ with $\left|E^{\prime}\right| \leq k$ such that $E^{\prime}$ contains at least one edge from every directed cycle in $G$ (equivalently, whether $G^{\prime}=\left(V, E \backslash E^{\prime}\right)$ is a directed acyclic graph (DAG)).

Now consider the problem of deciding whether there exists an $\alpha$ with $\ell(\alpha, \mu) \leq k$, and let $G_{\mu}$ denote the graph over the nodes associated with adjacency matrices $Y$, where $G_{\mu}$ has edge weights equal to the average $Y_{i j}^{\mu}=$ $\int Y_{i j} d \mu(Y)$. Since $\alpha$ induces an order of the nodes in this "expected" graph $G_{\mu}$, this is equivalent to finding an ordering of the nodes $i_{1}, \ldots, i_{n}$ (denoted $\left.i_{1} \prec i_{2} \prec \cdots \prec i_{n}\right)$ in $G_{\mu}$ such that the sum of the back edges is less than $k$,

$$
\sum_{i \succeq j} Y_{i j}^{\mu} \leq k
$$

Removing all the back edges (edges $(i \rightarrow j)$ in the expected graph $G_{\mu}$ such that $i \succeq j$ in the original ordering) leaves a DAG. Now, given a graph $G=(V, E)$, we can construct the expected graph $G_{\mu}$ directly from $G$ with weights $Y_{i j}^{\mu}=1$ if $(i \rightarrow j) \in E$ and 0 otherwise (set the probability that edge $(i \rightarrow j)$ appears to be $1 /|E|$ and set the associated $i j$ th adjacency matrix $Y$ to be $Y=0$ except that $\left.Y_{i j}=|E|\right)$. Then there is an $\alpha$ such that $\ell(\alpha, \mu) \leq k$ if and only if there is a feedback arc set $E^{\prime}$ with $\left|E^{\prime}\right| \leq k$.

10.2. Proof of Theorem 2. Before proceeding to the proof of Theorem 2 proper, we make a few remarks to reduce the space of functions $\phi$ that we must consider; we also argue that $h$ must be continuous and strictly increasing.

First, if the surrogate $\varphi$ from the definition (12) is structure-consistent, it must be classification calibrated: $\phi$ must be differentiable at 0 with $\phi^{\prime}(0)<$ 0 . This is a consequence of Bartlett, Jordan, and McAuliffe's analysis of binary classification and the correspondence between binary classification and pairwise ranking [1]. The same reasoning implies that we must have $h \geq 0$ on $\mathbb{R}_{+}$and $h$ strictly increasing. We now argue that $h$ must be continuous. Assume for the sake of contradiction that $h$ is discontinuous at some $c>0$, and without loss of generality assume that $h$ is not right continuous at $c$. Then there exists an $\epsilon>0$ such that $h(c+\delta) \geq h(c)+\epsilon$ for all $\delta>0$. Fix $\delta$ and $\gamma$ such that

$$
\gamma<\frac{\epsilon}{4 h(c)+2 \epsilon} \quad \text { and } \quad \delta<\frac{4 \gamma}{1-2 \gamma} c .
$$


Consider the case in which only two items are to be ranked, 1 and 2 , and let the preference $Y_{12}=c+\delta$ appear with probability $\frac{1}{2}-\gamma$ and $Y_{21}=c+\delta$ appear with probability $\frac{1}{2}+\gamma$ and $Y_{12}=c$. In this case,

$(1-2 \gamma) \delta<4 \gamma c$ or $\left(\frac{1}{2}-\gamma\right) \delta-\gamma c<\gamma c$, so $\left(\frac{1}{2}-\gamma\right)(c+\delta)<\left(\frac{1}{2}+\gamma\right) c$.

Any correct score assignment must thus rank item 1 before item 2, satisfying $\alpha_{1}>\alpha_{2}$. On the other hand, the condition on $\gamma, \epsilon$, and $h(c)$ implies $2 \gamma h(c)<$ $\left(\frac{1}{2}-\gamma\right) \epsilon$, so

$$
\left(\frac{1}{2}+\gamma\right) h(c)<\left(\frac{1}{2}-\gamma\right)(h(c)+\epsilon)<\left(\frac{1}{2}-\gamma\right) h(c+\delta)
$$

by assumption on the discontinuity of $h$. In particular, any minimizer of $\left(\frac{1}{2}+\gamma\right) h(c) \phi\left(\alpha_{1}-\alpha_{2}\right)+\left(\frac{1}{2}-\gamma\right) h(c+\delta) \phi\left(\alpha_{2}-\alpha_{1}\right)$ will in this case set $\alpha_{2}>\alpha_{1}$ since $\phi$ must be classification calibrated. In this case, the discontinuity of $h$ forces mis-ordering, and hence $h$ must be continuous.

The desired properties of $h$ established, consider the recession function [4] of $\phi$,

$$
\phi_{\infty}^{\prime}(d):=\sup _{t>0} \frac{\phi(t d)-\phi(0)}{t}=\lim _{t \rightarrow \infty} \frac{\phi(t d)-\phi(0)}{t} .
$$

For any $\phi$ bounded below with $\phi^{\prime}(0)<0$, we have that $\phi_{\infty}^{\prime}(1) \geq 0$. Throughout this analysis, we require the weaker assumption that $\phi$ decreases more slowly in the positive direction than the negative:

$$
\phi_{\infty}^{\prime}(1) \geq 0 \quad \text { or } \quad \phi_{\infty}^{\prime}(-1)=\infty .
$$

Consider two DAGs on nodes 1, 2, and 3 that induce only the four penalty values $Y_{12}, Y_{13}, Y_{23}$, and $Y_{31}$ (recall Figure 1). In this case, if $Y_{13}>Y_{31}$, any $\alpha$ minimizing $\ell(\alpha, \mu)$ must satisfy $\alpha_{1}>\alpha_{2}>\alpha_{3}$. We now show under some very general conditions that if $\varphi$ is edge-consistent, $\phi$ is non-convex.

Let $\phi^{\prime}(x)$ denote an element of the subgradient set $\partial \phi(x)$ and recall the definition $h_{i j}=\int h\left(Y_{i j}\right) d \mu$. The subgradient conditions for optimality of

$$
\ell_{\varphi}(\alpha, \mu)=h_{12} \phi\left(\alpha_{1}-\alpha_{2}\right)+h_{13} \phi\left(\alpha_{1}-\alpha_{3}\right)+h_{23} \phi\left(\alpha_{2}-\alpha_{3}\right)+h_{31} \phi\left(\alpha_{3}-\alpha_{1}\right)
$$

are that

$$
\begin{aligned}
& 0=h_{12} \phi^{\prime}\left(\alpha_{1}-\alpha_{2}\right)+h_{13} \phi^{\prime}\left(\alpha_{1}-\alpha_{3}\right)-h_{31} \phi^{\prime}\left(\alpha_{3}-\alpha_{1}\right) \\
& 0=-h_{12} \phi^{\prime}\left(\alpha_{1}-\alpha_{2}\right)+h_{23} \phi^{\prime}\left(\alpha_{2}-\alpha_{3}\right) .
\end{aligned}
$$

We begin by showing that whenever the condition (13) holds for $\phi$, there is a finite minimizer of $\ell_{\varphi}(\alpha, \mu)$ as defined by (14). The lemma is technical, so we provide its proof in Appendix 10.4. 
Lemma 12. Let the condition (13) hold and $\phi^{\prime}(0)<0$. There is a constant $C<\infty$ and a vector $\alpha^{*}$ minimizing $\ell_{\varphi}(\alpha, \mu)$ with $\left\|\alpha^{*}\right\|_{\infty} \leq C$.

The next lemma provides the essential lever we use to prove the theorem; we provide its proof in Appendix 10.5.

Lemma 13 (Inconsistency of convex losses). Suppose that $Y_{13}>Y_{31}>0$, $Y_{12}>0, Y_{23}>0$. Let

$\ell(\alpha, \mu)=Y_{12} 1\left(\alpha_{1} \leq \alpha_{2}\right)+Y_{13} 1\left(\alpha_{1} \leq \alpha_{3}\right)+Y_{23} 1\left(\alpha_{2} \leq \alpha_{3}\right)+Y_{31} 1\left(\alpha_{3}<\alpha_{1}\right)$

and $\ell_{\varphi}(\alpha, \mu)$ be defined as in Eq. (14). For convex $\phi$ with $\phi^{\prime}(0)<0$, we have

$$
\ell_{\varphi}^{*}(\mu)=\inf _{\alpha}\left\{\ell_{\varphi}(\alpha, \mu) \mid \alpha \notin \underset{\alpha^{\prime}}{\operatorname{argmin}} \ell\left(\alpha^{\prime}, \mu\right)\right\}
$$

whenever either of the following conditions is satisfied:

CONDition 1: $h_{23}<\frac{h_{31} h_{12}}{h_{13}+h_{12}}$ or CONDition 2: $h_{12}<\frac{h_{31} h_{23}}{h_{13}+h_{23}}$.

Lemma 13 allows us to construct scenarios under which arbitrary pairwise surrogate losses with convex $\phi$ are inconsistent. Indeed, assume for the sake of contradiction that $\varphi$ is structure-consistent. Recall that for $\phi$ convex, $\phi^{\prime}(0)<0$ by classification calibration. We will construct graphs $G_{1}$ and $G_{2}$ (with associated adjacency matrices $Y^{G_{1}}$ and $Y^{G_{2}}$ ) so that the resulting expected loss satisfies Condition 1 of Lemma 13 while additionally satisfying Definition 4's low-noise condition.

Consider the following two graphs:

$G_{1}=(\{1,2,3\},\{(1 \rightarrow 2),(1 \rightarrow 3)\}), \quad G_{2}=(\{1,2,3\},\{(2 \rightarrow 3),(3 \rightarrow 1)\})$.

Fix any weights $Y_{12}^{G_{1}}, Y_{13}^{G_{1}}, Y_{31}^{G_{2}}$ with $Y_{13}^{G_{1}}>Y_{12}^{G_{1}}>0$ and $Y_{13}^{G_{1}}>Y_{31}^{G_{2}}>0$, and let $\mu$ place half its mass on $G_{1}$ (or $Y^{G_{1}}$ ) and half its mass on $G_{2}$. As $h$ is continuous with $h(0)=0$, there exists some $\varepsilon>0$ such that $h(\varepsilon)<$ $2 h_{31} h_{12} /\left(h_{13}+h_{12}\right)$, where $h_{i j}=\frac{1}{2} h\left(Y_{i j}^{G_{1}}\right)+\frac{1}{2} h\left(Y_{i j}^{G_{2}}\right)$ as in the definition of the surrogate risk $\ell_{\varphi}$. Take $Y_{23}^{G_{2}}=\min \left\{\varepsilon,\left(Y_{13}^{G_{1}}-Y_{12}^{G_{1}}\right) / 2\right\}$. Then we have

$$
h_{23}=\frac{1}{2} h\left(Y_{23}^{G_{2}}\right) \leq h(\varepsilon) / 2<\frac{h_{31} h_{12}}{h_{13}+h_{12}} .
$$

Hence Condition 1 of Lemma 13 is satisfied, so $\varphi$ is not edge-consistent. Moreover, the fact that

$$
Y_{23}^{G_{2}} \leq \frac{Y_{13}^{G_{1}}-Y_{12}^{G_{1}}}{2}<Y_{13}^{G_{1}}-Y_{12}^{G_{1}}
$$

implies that the expected difference graph $G_{\mu}$ satisfies Definition 4. 
10.3. Proof of Theorem 3. Assume for the sake of contradiction that $\varphi$ is structure-consistent. As in the proof of Theorem 2, the function $h$ must be strictly increasing. Now let $c \in \mathbb{R}$ belong to the image $h((0, \infty))$ and define the shifted function $\widetilde{\phi}(x)=\phi(x-c)$. By the reduction to the binary case, $\widetilde{\phi}$ must be classification calibrated, whence $\widetilde{\phi}$ must be differentiable at 0 and satisfy $\widetilde{\phi}^{\prime}(0)<0$. Using a technique similar to that in our proof of Theorem 2 , we now construct a setting of four graphs and provide probabilities of appearance satisfying the conditions in Lemma 13 and Definition 4.

Consider the following four graphs on nodes $\{1,2,3\}$, each with one edge:

$$
\begin{aligned}
& G_{1}=(\{1,2,3\},\{(1 \rightarrow 2)\}), \quad G_{2}=(\{1,2,3\},\{(2 \rightarrow 3)\}), \\
& G_{3}=(\{1,2,3\},\{(1 \rightarrow 3)\}), \quad G_{4}=(\{1,2,3\},\{(3 \rightarrow 1)\}) .
\end{aligned}
$$

Choose constant edge weights $Y_{12}^{G_{1}}=Y_{13}^{G_{2}}=Y_{23}^{G_{3}}=Y_{31}^{G_{4}}=h^{-1}(c)>0(h$ is increasing and thus invertible), and set the probabilities of appearance to be $\mu \equiv(.25, .01, .5, .24)$. Recalling $\widetilde{\phi}$, we have

$$
\begin{aligned}
\ell_{\varphi}(\alpha, \mu)= & \mu\left(G_{1}\right) \widetilde{\phi}\left(\alpha_{1}-\alpha_{2}\right)+\mu\left(G_{2}\right) \widetilde{\phi}\left(\alpha_{2}-\alpha_{3}\right) \\
& +\mu\left(G_{3}\right) \widetilde{\phi}\left(\alpha_{1}-\alpha_{3}\right)+\mu\left(G_{4}\right) \widetilde{\phi}\left(\alpha_{3}-\alpha_{1}\right) .
\end{aligned}
$$

Notably, $\widetilde{\phi}$ is convex and satisfies the recession condition (13). Moreover

$Y_{13}-Y_{31}=h^{-1}(c)\left(\mu\left(G_{3}\right)-\mu\left(G_{4}\right)\right) \geq h^{-1}(c)\left(\mu\left(G_{1}\right)+\mu\left(G_{2}\right)\right)=Y_{12}+Y_{23}>0$,

so $G_{\mu}$ is a DAG satisfying the low-noise condition. However, the probabilities $\mu$ satisfiy

$$
\mu\left(G_{2}\right)<\frac{\mu\left(G_{4}\right) \mu\left(G_{1}\right)}{\mu\left(G_{3}\right)+\mu\left(G_{1}\right)} .
$$

Hence, by Lemma 13, we have the contradiction that

$$
\ell_{\varphi}^{*}(\mu)=\inf _{\alpha}\left\{\ell_{\varphi}(\alpha, \mu) \mid \alpha \notin \underset{\alpha^{\prime}}{\operatorname{argmin}} \ell(\alpha, \mu)\right\} .
$$

10.4. Proof of Lemma 12. Let $\left\{\alpha^{(n)}\right\}_{n=1}^{\infty} \subset \mathbb{R}^{3}$ be a sequence satisfying $\ell_{\varphi}\left(\alpha^{(n)}, \mu\right) \rightarrow \ell_{\varphi}^{*}(\mu)$. Suppose for the sake of contradiction that $\lim \sup _{n}\left(\alpha_{i}^{(n)}-\right.$ $\left.\alpha_{j}^{(n)}\right)=\infty$ for some $i, j$. The convexity of $\phi$ coupled with $\phi^{\prime}(0)<0$ imply that $\lim \sup _{n} \phi\left(\alpha_{j}^{(n)}-\alpha_{i}^{(n)}\right)=\infty$; the recession condition (13) (that $\phi_{\infty}^{\prime}(1) \geq 0$ or $\left.\phi_{\infty}^{\prime}(-1)=\infty\right)$ guarantees $\lim \sup _{n} \ell_{\varphi}\left(\alpha^{(n)}, \mu\right)=\infty$ whenever $i>j$ or $i=1$ and $j=3$. We thus have two remaining cases:
(a) $\limsup _{n}\left(\alpha_{1}^{(n)}-\alpha_{2}^{(n)}\right)=\infty$ or
(b) $\limsup _{n}\left(\alpha_{2}^{(n)}-\alpha_{3}^{(n)}\right)=\infty$ 
In case (a), we note that $\alpha_{1}^{(n)}-\alpha_{2}^{(n)}=\alpha_{1}^{(n)}-\alpha_{3}^{(n)}+\alpha_{3}^{(n)}-\alpha_{2}^{(n)}$, but there must exist a constant $C$ such that $\left|\alpha_{1}^{(n)}-\alpha_{3}^{(n)}\right| \leq C$ for all $n$ by our earlier argument. This would imply that $\lim \sup _{n}\left(\alpha_{3}^{(n)}-\alpha_{2}^{(n)}\right)=\infty$, a contradiction. Similarly, for case (b), we may use $\alpha_{2}^{(n)}-\alpha_{3}^{(n)}=\alpha_{2}^{(n)}-\alpha_{1}^{(n)}+\alpha_{1}^{(n)}-\alpha_{3}^{(n)}$ and our ealier argument that $\left|\alpha_{1}^{(n)}-\alpha_{3}^{(n)}\right| \leq C$ for all $n$ to see that case (b) would require $\lim \sup _{n}\left(\alpha_{2}^{(n)}-\alpha_{1}^{(n)}\right)=\infty$, another contradiction.

As a consequence, there must be some $C<\infty$ with $\left|\alpha_{i}^{(n)}-\alpha_{j}^{(n)}\right| \leq C$ for all $i, j, n$. The conditional surrogate risk $\ell_{\varphi}(\alpha, \mu)$ is shift invariant with respect to $\alpha$, so without loss of generality we may assume $\alpha_{3}^{(n)}=0$, and thus $\left|\alpha_{i}^{(n)}\right| \leq C$. Convex functions are continuous on compact domains [4, Chapter IV.3], and thus some $\alpha$ with $\|\alpha\|_{\infty} \leq C$ attains the infimum

$$
\inf _{\|\alpha\|_{\infty} \leq C} \ell_{\varphi}(\alpha, \mu)=\ell_{\varphi}^{*}(\alpha, \mu) .
$$

10.5. Proof of Lemma 13. Lemma 12 shows that the optimal $\ell_{\varphi}^{*}(\mu)$ is attained by some finite $\alpha$. Thus, we fix an $\alpha^{*}$ satisfying the optimality condition (15) and let $\delta_{i j}=\alpha_{i}^{*}-\alpha_{j}^{*}$ and $g_{i j}=\phi^{\prime}\left(\delta_{i j}\right)$ for $i \neq j$. We make use of the monotonicity of subgradients, that is, $\delta_{i j}>\delta_{k l}$ implies $g_{i j} \geq g_{k l}$ [e.g., 4 , Chapter VI]. By condition (15),

$$
\begin{aligned}
& g_{13}-g_{12}=\frac{h_{31}}{h_{13}} g_{31}-\left(1+\frac{h_{12}}{h_{13}}\right) g_{12} \\
& g_{13}-g_{23}=\frac{h_{31}}{h_{13}} g_{31}-\left(1+\frac{h_{23}}{h_{13}}\right) g_{23} .
\end{aligned}
$$

Suppose for the sake of contradiction that $\alpha^{*} \in \operatorname{argmin}_{\alpha} \ell(\alpha, \mu)$. As $\delta_{13}=$ $\delta_{12}+\delta_{23}$, we have that $\delta_{13}>\delta_{12}$ and $\delta_{13}>\delta_{23}$. The convexity of $\phi$ implies that if $\delta_{13}>\delta_{12}$, then $g_{13} \geq g_{12}$. If $g_{12} \geq 0$, we thus have that $g_{13} \geq 0$ and by (15), $g_{31} \geq 0$. This is a contradiction since $\delta_{31}<0$ gives $g_{31} \leq \phi^{\prime}(0)<0$. Hence, $g_{12}<0$. By identical reasoning, we also have that $g_{23}<0$.

Now, $\delta_{23}>0>\delta_{31}$ implies that $g_{23} \geq g_{31}$, which combined with the equality (16a) and the fact that $g_{23}=\left(h_{12} / h_{23}\right) g_{12}$ (by the first-order optimality equation (15)) gives

$$
g_{13}-g_{12} \leq \frac{h_{31}}{h_{13}} g_{23}-\left(1+\frac{h_{12}}{h_{13}}\right) g_{12}=\left(\frac{h_{31} h_{12}}{h_{23}}-h_{13}-h_{12}\right) \frac{g_{12}}{h_{13}} .
$$

Since $g_{12} / h_{13}<0$, we have that $g_{13}-g_{12}<0$ whenever $h_{31} h_{12} / h_{23}>$ $h_{13}+h_{12}$. But when $\delta_{13}>\delta_{12}$, we must have $g_{13} \geq g_{12}$, which yields a contradiction under CONDITION 1. 
Similarly, $\delta_{12}>0>\delta_{31}$ implies that $g_{12} \geq g_{31}$, which with $g_{12}=$ $\left(h_{23} / h_{12}\right) g_{23}$ and equality $(16 \mathrm{~b})$ yields

$$
g_{13}-g_{23} \leq \frac{h_{31}}{h_{13}} g_{12}-\left(1+\frac{h_{23}}{h_{13}}\right) g_{23}=\left(\frac{h_{31} h_{23}}{h_{12}}-h_{13}-h_{23}\right) \frac{g_{23}}{h_{13}} .
$$

Since $g_{23} / h_{13}<0$, we further have that $g_{13}-g_{23}<0$ whenever $h_{31} h_{23} / h_{12}>$ $h_{13}+h_{23}$. This contradicts $\delta_{13}>\delta_{23}$ under Condition 2 .

\section{References.}

[1] P. L. Bartlett, M. I. Jordan, and J. McAuliffe. Convexity, classification, and risk bounds. Journal of the American Statistical Association, 101:138-156, 2006.

[2] P. Billingsley. Convergence of Probability Measures. Wiley, Second edition, 1999.

[3] J. C. Duchi, L. Mackey, and M. I. Jordan. On the consistency of ranking algorithms. In Proceedings of the 27th International Conference on Machine Learning, 2010.

[4] J. Hiriart-Urruty and C. Lemaréchal. Convex Analysis and Minimization Algorithms I $\&$ II. Springer, New York, 1996.

[5] R. M. Karp. Reducibility among combinatorial problems. In Complexity of Computer Computations, pages 85-103. Plenum Press, 1972.

[6] C. McDiarmid. On the method of bounded differences. Surveys in Combinatorics, pages 148-188, 1989.

[7] R. T. Rockafellar and R. J. B. Wets. Variational Analysis. Springer, New York, 1998.

[8] I. Steinwart. How to compare different loss functions. Constructive Approximation, 26:225-287, 2007.

[9] A. Tewari and P. L. Bartlett. On the consistency of multiclass classification methods. Journal of Machine Learning Research, 8:1007-1025, 2007.

[10] A. W. van der Vaart and J. A. Wellner. Weak Convergence and Empirical Processes: With Applications to Statistics. Springer, New York, 1996.

[11] T. Zhang. Statistical analysis of some multi-category large margin classification methods. Journal of Machine Learning Research, 5:1225-1251, 2004.

Departments of EECS and Statistics

University of California, Berkeley

E-MAIL: jduchi@cs.berkeley.edu jordan@stat.berkeley.edu
Department of Statistics

STANFORD UNIVERSITY

E-MAIL: lmackey@stanford.edu 